\title{
Review
}

\section{BAG3: a multifaceted protein that regulates major cell pathways}

\author{
A Rosati $^{1,2}$, V Graziano ${ }^{3}$, V De Laurenzi ${ }^{2,3}$, M Pascale $^{1,2}$ and MC Turco ${ }^{*, 1,2}$
}

Bcl2-associated athanogene 3 (BAG3) protein is a member of BAG family of co-chaperones that interacts with the ATPase domain of the heat shock protein (Hsp) 70 through BAG domain (110-124 amino acids). BAG3 is the only member of the family to be induced by stressful stimuli, mainly through the activity of heat shock factor 1 on bag3 gene promoter. In addition to the BAG domain, BAG3 contains also a WW domain and a proline-rich (PXXP) repeat, that mediate binding to partners different from Hsp70. These multifaceted interactions underlie BAG3 ability to modulate major biological processes, that is, apoptosis, development, cytoskeleton organization and autophagy, thereby mediating cell adaptive responses to stressful stimuli. In normal cells, BAG3 is constitutively present in a very few cell types, including cardiomyocytes and skeletal muscle cells, in which the protein appears to contribute to cell resistance to mechanical stress. A growing body of evidence indicate that BAG3 is instead expressed in several tumor types. In different tumor contexts, BAG3 protein was reported to sustain cell survival, resistance to therapy, and/or motility and metastatization. In some tumor types, down-modulation of BAG3 levels was shown, as a proof-of-principle, to inhibit neoplastic cell growth in animal models. This review attempts to outline the emerging mechanisms that can underlie some of the biological activities of the protein, focusing on implications in tumor progression.

Cell Death and Disease (2011) 2, e141; doi:10.1038/cddis.2011.24; published online 7 April 2011

Subject Category: Cancer

Bcl2-associated athanogene (BAG) proteins are a family of co-chaperones that interact with the ATPase domain of the heat shock protein (Hsp) 70 through a specific structural domain known as BAG domain (110-124 amino acids)., ${ }^{1,2}$ Members of the family are found throughout the evolution in yeast (Saccharomyces cerevisiae, Schizosaccharomyces pombe), invertebrates (Caenorhabditis elegans, Ciona intestinalis, Drosophila), amphibians (Xenopus laevis), mammals (humans, mice) and plants (Oryza sativa, Arabidopsis thaliana), ${ }^{1,3-6}$ suggesting a fundamental biological role.

BAG3 was originally identified by yeast two-hybrid screening using the ATPase domain of the Hsp (heat shock cognate (Hsc)/Hsp) 70 as a bait. ${ }^{1}$ In addition to the BAG domain, BAG3 contains a WW domain and a proline-rich repeat (PXXP; Figure 1a) that mediate binding to other partners. ${ }^{1,7,8}$

Here we will outline the roles so far identified of BAG3 in the regulation of major biological processes, that is, apoptosis, development, cytoskeleton organization and autophagy. By modulating these pathways, BAG3 appears to mediate cell adaptive responses to stressful stimuli, and its alterations result in altered homeostasis and reduced cytoprotection, explaining why its expression is often found deregulated in a vast series of tumors.

\section{BAG3 Protein and bag3 Gene Expression}

Two BAG3 forms have been described so far: one is the full-lenght product of the bag3 gene having an apparent mass of $74 \mathrm{kDa}$, the other one is a shorter BAG3 protein found in association to neuronal synaptosomes ${ }^{2,9-11}$ (Figure 1b). The BAG3 full-length protein is localized in the cytoplasm, mainly concentrated in the rough endoplasmic reticulum; on cell exposure to stressors, a slightly different molecular weight variant of this form can also be observed, and both co-exist in some cell types and run as a doublet in a standard western blot. The origin of this doublet is currently unknown, but it could derive from post-translational modifications as phosphorylations, indeed BAG3 protein contains several serinerich motifs and 10 tyrosine residues. Tyrosine phosphorylation of BAG3 occurs on EGF stimulation in human breast cancer cell lines. ${ }^{12}$ This post-translational variant of BAG3 protein appears also to be associated to Bruton's tyrosine kinase (Btk) protein in Defew cells on oxidative stress induction. ${ }^{13}$ Recently, a shorter form of BAG3 $(40 \mathrm{kDa})$ has been characterized by immunoprecipitation from neural synaptosomes homogenates and successive mass spectrometry, ${ }^{11}$ again it is yet unknown whether this form derives from the alternative splicing or proteolytic processing.

\footnotetext{
${ }^{1}$ Department of Pharmaceutical and Biomedical Sciences (FARMABIOMED), University of Salerno, Fisciano, Italy; ${ }^{2}$ BIOUNIVERSA srl, Fisciano, Italy and ${ }^{3}$ Department of Biomedical Sciences, University of Chieti-Pescara, Chieti, Italy

*Corresponding author: MC Turco, Department of Pharmaceutical Sciences, Division of Biomedicine 'Arturo Leone', University of Salerno, via ponte don Melillo, Fisciano 84084, Italy. Tel: + 3908996 9774; Fax: + 39089969 602; E-mail: mcturco@ unisa.it

Keywords: BAG3; cancer; apoptosis; autophagy; cytoskeleton

Abbreviations: BAG, Bcl2-associated athanogene

Received 21.2.11; accepted 23.2.11; Edited by RA Knight
} 
a

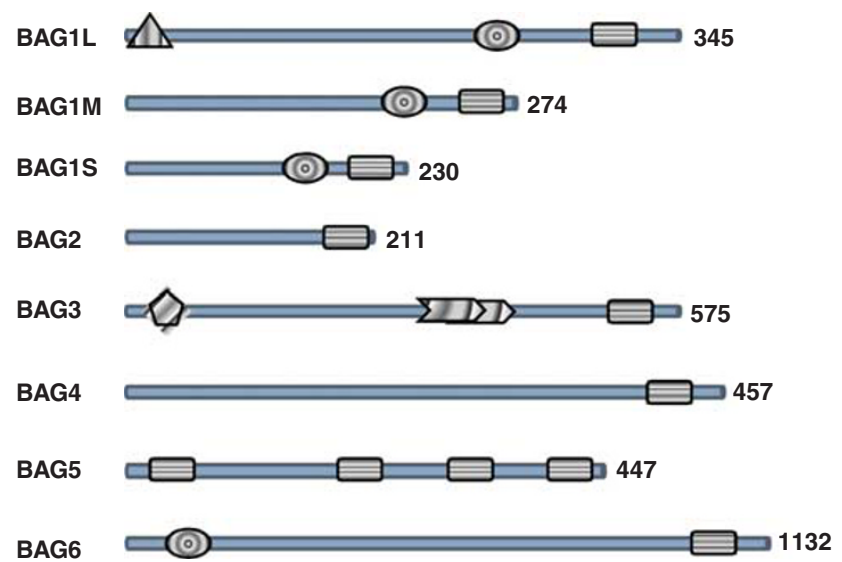

b

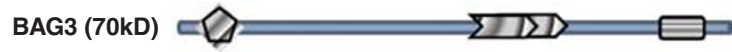

BAG3 (40kD) $-\triangle D D D$

\section{BAG $\triangle$ NLS $\emptyset$ WW (D) $\sum D D$ PXXP repeats}

Figure 1 (a) Human BAG family proteins. All six reported BAG proteins contain a BAG domain at their $\mathrm{C}$-terminus. Some BAG proteins contain other domains including nuclear localization signal (NLS), ubiquitin-like (UBL) domain, WW domain and proline-rich regions (PXXP). Numbers next to the linear peptide sequence indicate amino acid number in the proteins. (b) Schematic representation of the full-length BAG3 protein and the shorter BAG3 protein found to be associated to synaptosomes

BAG3 expression has also been observed in the nuclei of some cell types, including pancreatic carcinoma cells (unpublished results from our laboratory). Interestingly, BAG3 was shown to localize in nuclei of cells infected by some viruses; those findings suggest an involvement of BAG3 in viral expression and/or replication, and indeed some reports indicate a requirement for BAG3 for virus gene expression and/or viral progeny production. ${ }^{12,14-18}$ In keeping with those results, viral infection was shown to induce the expression of BAG3, as a possible tool contributing to virus life cycle. ${ }^{12,19}$

In humans, bag3 gene expression is constitutive in myocytes, a few other normal cell types and several primary tumors or tumor cell lines (lymphoid or myeloid leukemias, lymphomas, myeloma, neuroblastoma, pancreas, thyroid, breast and prostate carcinomas, melanoma, osteosarcoma, kidney, colon and ovary cancers, glioblastoma). ${ }^{20-37}$ It is instead induced in different normal cell types (leukocytes, epithelial and glial cells, retinal cells) by a variety of stressors, such as oxidants, high temperature, serum deprivation, heavy metals, HIV-1 infection, ELF exposure, electrophile stress, hemodialysis treatment, pulsed ultrasound, retinal light damage, kanaic acid-induced seizure and transient forebrain ischemia. 2,10,14,19,35,37-44

The expression of stress-responsive genes is regulated by the heat shock transcription factors (HSFs) ${ }^{45}$ including HSF1, that is required for tumor initiation and maintenance in a variety of cancer models. ${ }^{46}$ bag3 gene promoter activity is regulated by $\mathrm{HSF} 1,{ }^{31,47,48}$ again suggesting a role in tumor formation. Other known transcription factors that are known to regulate bag3 expression are Egr1 ${ }^{29} \mathrm{AibZIP}^{26}$ and WT1. ${ }^{49}$ The regulation by WT1 is of particular interest because of the role played by this transcription factor in human leukemias ${ }^{50}$ (see the section BAG3 in leukemias). Furthermore, in some cell types bag3 expression seems to be controlled by its own product. $^{51}$

A number of drugs - proteasome inhibitors, TNF-related apoptosis-inducing ligand, fludarabine, cytosine arabinoside and etoposide - increase BAG3 protein levels, this in turn contributes to cell resistance to therapy, and indeed bag3 silencing improves neoplastic cell apoptotic response to drugs. $^{21,22,24,30,37,52,53}$

\section{BAG3 in Development}

In some cell types, bag3 expression appears to be developmentally regulated. In the developing central nervous system (CNS) of a rat, there is a transient expression, detectable by immunohistochemistry, of BAG3 in the cerebral cortex and hippocampus, whereas a considerable expression is maintained in the rostral migratory stream and the subventricular zone of the lateral ventricle; there is an abrupt increase of BAG3-positive neurons in the cortex and hippocampus during the first postnatal week, which declines thereafter. Two specific populations of BAG3-positive neurons can be identified in the developing forebrain of a rat. $^{54}$ Furthermore, recent results indicated that BAG3 is expressed in neural progenitors and sustains proliferation, mainly in response to FGF2, in those cells. ${ }^{55}$ In addition, an early transient expression of BAG3 was observed in midline radial glia in the developing brainstem and spinal cord of a rat. ${ }^{56}$ Together, these pieces of evidence indicate a role for BAG3 in the development of both the neuronal and glial components of central nervous system. In agreement with the hypothesis of BAG3 involvement in CNS development, an altered BAG3 expression was observed in the cerebellum of hypothyroid juvenile mice, and was suggested to contribute to impaired development of the hypothyroid brain. Moreover, in rat and human cardiomyocytes, BAG3 protein appears to be expressed during differentiation from cardiomyoblasts and to sustain myogenin expression. ${ }^{57}$ These findings indicate an involvement of BAG3 protein in late heart development and are in keeping with the role of BAG3 in the survival and myofibrillar integrity in cardiocytes and, in general, in muscle cells. Indeed, mice with homozygous disruption of bag3 gene develop post birth an early fulminant myopathy ${ }^{23}$ and, in humans, a heterozygous p.Pro209Leu mutation in BAG3 protein was recently recognized to be responsible for a severe muscular dystrophy with cardiomyopathy and severe respiratory insufficiency. ${ }^{57-59}$ In one of the three studied families carrying this mutation, an axonal neuropathy was also present. This observation is intriguing in view of the reported localization of a $40 \mathrm{kD}$ form of BAG3 in synaptosomes ${ }^{11}$ and of the above discussed role of BAG3 in CNS. Finally, a recent paper reports a role of BAG3 in the development of the hematopoietic system, showing that mice with a targeted disruption of bag3 exhibit a loss of hematopoietic stem cells 
and defective B-cell development, because of the microenvironmental defect, that is, an alteration in the vascular stem cell niche. ${ }^{60}$ The authors observed a defective growth of stromal progenitor cells in colony-forming unit fibroblasts, a defect in sinusoidal endothelium, and the loss of stromal cells expressing CXCL-12 or IL-7 in the bone marrow. ${ }^{60}$ The molecular mechanisms underlying those perturbations could, once identified, disclose novel prospects in the understanding of the hematopoietic process.

Last but not least, recent evidence indicate that BAG3 is also implicated in regulating the general metabolic state of the organism, as bag3-deficient mice were reported to show significant hypoglycemia, a decrease in triglyceride and cholesterol levels and growth retardation, and died by 3 weeks after birth. ${ }^{61}$

Altogether these data show a fundamental role of BAG3 in regulating essential physiological events.

\section{BAG3 and Apoptosis}

A number of studies in tumor cell lines of different origin have shown that bag3 silencing or hyperexpression results in, respectively, enhancing or inhibiting spontaneous or drug-induced apoptosis. 9,24,25,30,31,34,36,37,44,49 Furthermore, caspases trigger BAG3 cleavage, thereby facilitating the apoptotic process. ${ }^{52,62}$

BAG3 seems to influence cell survival by interacting with different molecular partner, thus activating multiple pathways. A first demonstrated mechanism of BAG3 anti-apoptotic activity is mediated by its role, as a co-chaperone, in protein delivery to the proteasome. Indeed, although another member of BAG family, that is, BAG1, positively cooperates with Hsp70 and CHIP (C-terminus of the Hsc70-interacting protein) to direct, through its ubiquitin-like domain (Figure 1), client proteins to proteasome, ${ }^{63}$ BAG3 can interfere with this process by competing with BAG1.2,4,29,52 Indeed, in osteosarcoma and melanoma cells, BAG3 protects IKK- $\gamma$ from proteasome delivery and this results in sustained NF-kB activation and cell survival. ${ }^{37} \mathrm{~A}$ different mechanism has been observed in glioblastoma cells, in which BAG3 retains BAX protein in the cytosol, preventing its mitochondrial translocation. ${ }^{36}$ Both mechanisms rely on an interaction between BAG3 and $\mathrm{Hsp} 70{ }^{36,37}$ We can speculate that through its binding to Hsp70, BAG3 might also positively or negatively modulate folding of other apoptosis-regulating proteins, and expect that future research will disclose a very complex regulative mechanism mediated by this protein. More over, as HSP70 can bind to $\mathrm{AU}$-rich elements in the $3^{\prime}$-untranslated regions, regulating expression of a number of proteins, including the well-known pro-apoptotic, BH3 only protein Bim, ${ }^{64}$ BAG3 might be expected to regulate $\mathrm{Hsp} 70$ ability to stabilize Bim mRNA and possibly other mRNAs, involved in various cell functions. Finally, we can envisage functions of BAG3 that are independent of $\mathrm{Hsp} 70$, as it could also bind some client proteins through its WW or PXXP domain, directly influencing their stability, localization or activity (Figure 2). It is likely that the availability of the different partners underlies the different mechanisms through which BAG3 exerts its anti-apoptotic activity in different cell types.

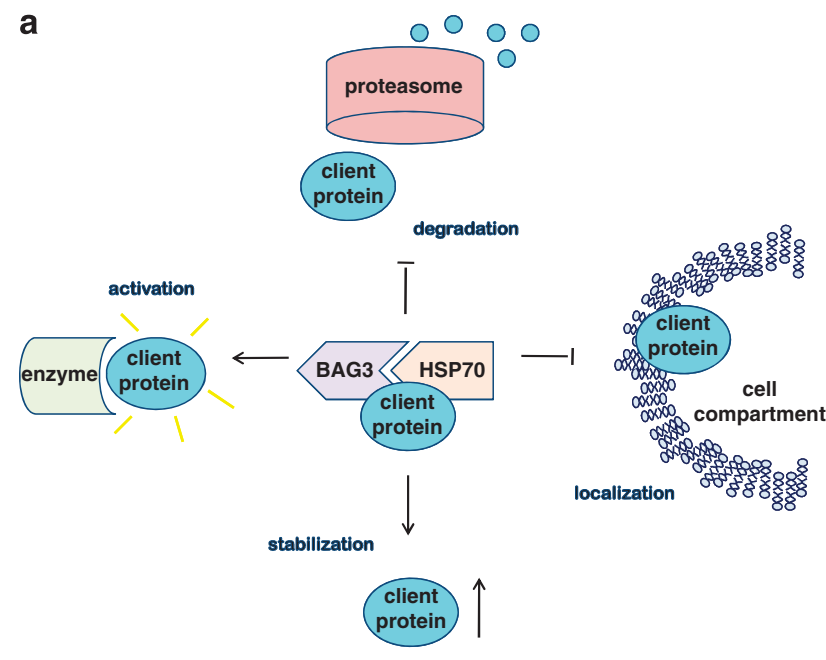

b

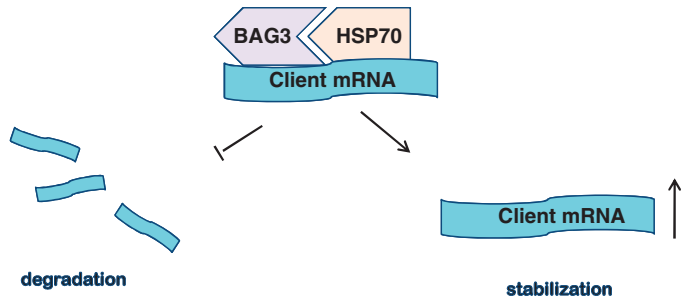

Figure 2 Mechanism of BAG3 activity on (a) client proteins, (b) client mRNAs

\section{BAG3, Cytoskeleton, Cell Adhesion and Motility}

It has been shown that bag3 silencing reduces adhesion and/or motility of epithelial (breast, prostate) tumor cells. ${ }^{27,32,65,66}$ In MDA435 human breast cancer cells, BAG3 overexpression resulted in a decrease in migration and adhesion to matrix molecules; the decrease was reversed on deletion of the BAG3 proline-rich (PXXP) domain, indicating that an interaction of BAG3 with a $\mathrm{SH}_{3}$ domain-containing protein was involved. ${ }^{65}$ Expression array studies showed that indeed BAG3 regulated, in a PXXP-dependent manner, the expression of CCN (Cyr61, connective tissue growth, NOV) 1, a matricellular signaling protein that promotes cell adhesion through integrins and heparan sulfate-containing proteoglycans. $^{32}$ In addition, BAG3 seems to regulate cell adhesion through binding to guanine nucleotide exchange factor 2 (PDZGEF2). This protein induces the activation of Rap1, a regulator of cell-cell junction formation and remodeling, and increases integrin-mediated cell adhesion. The PPDY motif at the C-terminus of PDZGEF2 was shown to bind to the WW domain of BAG3, and PDZGEF2 knockdown reduced BAG3 ability to induce cell adhesion in Cos7 cells. ${ }^{67}$

Similarly to what we described above for apoptosis, the ability of BAG3 to regulate cell adhesion appears to rely on multiple interactions of this protein through different structural domains. It is important to note that modulation of cell adhesion has profound effects on cell vitality, as cell detachment from matrix induces the apoptotic process known as anoikis. ${ }^{68}$ Therefore, BAG3 alterations could underlie effects on both cell detachment/motility and resistance to associated anoikis, thereby favouring metastatization. 
Finally, we recently reported that BAG3, through its interaction with the cytosolic chaperonin CCT (chaperonin containing TCP-1), regulates actin folding. ${ }^{66}$ This property of $\bar{B} A G 3$ highlights its involvement in cytoskeleton organization, possibly influencing not only cell survival and migration, but also membrane trafficking and organellar dynamics.

\section{BAG3 and Autophagy}

The role played by BAG3 in the cytoskeleton remodeling and membrane trafficking suggests the possibility that it might be involved in autophagy. With this term, we refer to a set of nonspecific bulk degradation processes, in which cells deliver cytoplasmic substrates for lysosomal degradation. ${ }^{69}$ There is a chaperone-mediated autophagy (CMA), selective for cytosolic proteins that contain a pentapeptide motif: this motif is recognized by the chaperone Hsc70, which transfers protein substrates to lysosomes. ${ }^{69}$ As BAG3 is a Hsc/Hsp 70 co-chaperone, it is plausible to imagine its involvement in CMA, but we can also envisage a role in the other two types of autophagy, namely micro- and macroautophagy. In macroautophagy, cells form double-membrane vesicles, called autophagosomes, around a portion of cytoplasm; autophagosomes are trafficked along microtubules and fused with lysosomes, resulting in degradation of their contents. Microautophagy is a process in which lysosomes directly engulf cytoplasm. $^{69}$ Similar to Hsps and BAG3, autophagy is upregulated under stress. Indeed, the autophagic process is important in promoting cell survival in several conditions, such as protein aggregate formation, nutrient and growth factor deprivation, ER stress and pathogen infection. Defective autophagy is associated with diverse diseases, including neurodegeneration, lysosomal storage diseases, muscular dystrophies, cancers and Crohn's disease. ${ }^{69}$

BAG3 participates, along with HspB8, a member of the $\mathrm{HspB}$ family of molecular chaperones, in the degradation of misfolded and aggregated proteins via macroautophagy. Indeed HspB8 forms a stable complex with BAG3 in cells and the formation of this complex is essential for the HspB8mediated degradation of the polyglutamine protein $\mathrm{Htt} 43 \mathrm{Q}$ (HunTingTin exon 1 fragment with 43 CAG repeats), a pathogenic form of huntingtin prone to aggregation. ${ }^{70,71}$ HspB8 and BAG3 induce, in a Hsp70-independent manner, the phosphorylation of the $\alpha$-subunit of the translation initiator factor elF2; this in turn causes a translational shutdown and stimulates autophagy. The mechanism by which the BAG3/ HspB8 complex induces elF2 phosphorylation is not completely understood. ${ }^{70,71}$ BAG3 binding to HspB8 is mediated by two conserved Ile-Pro-Val (IPV) motifs located between the $\mathrm{W}$ - and the Pro-rich domains of the co-chaperone; deletion of these motifs suppresses HspB8 activity in Htt43Q degradation. $^{72}$ Interestingly, the p.Pro209Leu mutation responsible for dystrophy is in one of the two IPV motifs. ${ }^{58,72}$ This suggests that the disease pathogenesis could involve defective autophagy. Through the same protein region, BAG3 can bind also to another chaperone HspB6. This is particularly intriguing in view of the cardioprotective property of HspB6/ Hsp20 ${ }^{73}$ and its role in myocyte contractility. ${ }^{74}$ Finally, BAG3 complexes with the ubiquitin-binding protein p62/SQSTM1, that, by binding simultaneously the autophagosome protein
LC3 and polyUb-proteins, controls the sequestration of polyUb-proteins into inclusion bodies for autophagic degradation. ${ }^{75}$

Therefore, BAG3 appears to negatively regulate protein delivery to proteasome, by competing with BAG1, but at the same time it stimulates autophagy. This suggests that, although BAG1 constitutively assures, in combination with Hsc/Hsp70, proteostasis through the ubiquitin-proteasome system (UPS), stressful stimuli, by inducing BAG3 increase, determine an inhibition of UPS (because of the BAG3 competition with BAG1) and a stimulation of autophagy. In agreement with this hypothesis, BAG3/BAG1 protein ratio increases, concomitantly with an increase in autophagy, in an in vitro model of cell aging (replicative senescence: 190 cells) and in rodent brain neurons during aging. ${ }^{75}$ The authors attribute BAG3 increase to oxidative stress due to the enhanced pro-oxidant milieu characteristic of aging. As this milieu enhances the occurrence of protein aggregation, BAG3-stimulated autophagy is interpreted as an adaptive response of the protein quality control system. ${ }^{75}$

\section{BAG3 in Leukemias}

BAG3 is expressed at low levels in normal blood cells, whereas it is highly expressed both in primary leukemic blasts or established cells lines from leukemic patients. ${ }^{21,22}$ Furthermore, its levels are markedly higher in drug-resistant patients compared with patients that resulted responsive to chemotherapy. One mechanism responsible for bag3 overexpression in leukemic cells relies on the effect of the WT1 transcription factor on the bag 3 gene promoter. ${ }^{49}$ WT1 is overexpressed in acute lymphoblastic and myeloblastic leukemia, and high levels of the protein are associated with a poor response to therapy. ${ }^{50}$ We recently showed that the WT1 isoform + KTS was able to positively regulates BAG3 expression by a transcriptional mechanism, via a direct binding on two WT1 consensus sites on the bag3 gene promoter. WT1 knockdown induces apoptosis, whereas its overexpression protects leukemic cells from apoptosis inducers; these effect are in part because of the modulation of BAG3 protein levels. ${ }^{49}$ The role of BAG3 modulation is even more evident in primary human leukemia cells. Indeed in leukemic cells from 24 patients affected by B-cell chronic lymphocytic leukemia (B-CLL) ${ }^{21}$ and from 11 children affected by acute lymphoblastic leukemia, ${ }^{22}$ we observed that BAG3 down-modulation by specific antisense oligodeoxynucleotides results in enhancing the percentage of apoptotic elements by $>100 \%$. Cell apoptosis was enhanced in cells either untreated or incubated with chemotherapeutic drugs. Those were the first reported evidence of the apoptosisregulating activity of BAG3 protein in primary tumors. ${ }^{21,22}$

We also showed that BAG3 down-modulation increased apoptosis of primary normal human peripheral blood mononuclear cells as well as of leukemic cells incubated with agents able to induced oxidative stress. ${ }^{39}$ Furthermore, a physical interaction was observed between BAG3 and the Btk under oxidative stress conditions. All those evidences assign to BAG3 a critical role in the leukemic cell survival and responsiveness to chemotherapy and should prompt 
investigators to further investigate its role in these deseases and the possibility of exploiting it as a molecular target.

\section{Conclusions}

From the first evidence of BAG3-mediated survival in $\mathrm{B}-\mathrm{CLLs},{ }^{21}$ a number of reports confirmed the anti-apoptotic activity of the protein in tumors of the hematopoietic $^{2,13,21,22,25,28,30,39,40,49}$ and other $2,20,24,25,29,31,34,36,37,44$ compartments. Two principal elements contribute to the role of BAG3 in vast range of tumors: bag3 induction by $\mathrm{HSF}_{1},{ }^{47}$ a transcription factor activated in many cancer types; ${ }^{46}$ and BAG3 ability to sustain of NF- $\kappa$ B activity. ${ }^{76}$ In addition, BAG3, because of its multifaceted ability to complex with many proteins, can regulate factors that are particularly relevant in the context of specific tumor types, such as BAX in glioblastoma cells. ${ }^{36}$

Overall, studies on BAG3 expression and activity in normal and neoplastic cells, and of its regulation during the development, increasingly delineate a complex picture of BAG3 involvement in physiological and pathological processes. The pleiotropic functions attributed to BAG3 probably reflect the adapter nature of this multidomain (BAG, WW and PXXP) protein and its ability to assemble scaffolding complexes, which participate in more than one signal transduction pathway.

It is of particular interest that BAG3 deficiency can result in the loss of hematopoietic stem cells due to a defect in stem cell niche. ${ }^{61}$ Therefore, BAG3 is required for the stem cellsupporting activity of microenvironmental cells. This finding suggests that BAG3 may also regulate the cancer stem cell-supporting activity of stromal cells in the hematopoietic and possibly in other compartments. Again, NF- $\kappa \mathrm{B}$ factors could represent key targets of BAG3, not only for the expression of tumor cell genes that sustain cell survival, but also for the expression of stromal cells' soluble and cell surface molecules that mediate bidirectional interactions between tumor cells and their environment. ${ }^{77,78}$

\section{Conflict of interest}

The authors declare no conflict of interest.

1. Takayama S, Xie Z, Reed JC. An evolutionarily conserved family of $\mathrm{Hsp} 70 / \mathrm{Hsc70}$ molecular chaperone regulators. J Biol Chem 1999; 274: 781-786.

2. Rosati A, Ammirante M, Gentilella A, Basile A, Festa M, Pascale M et al. Apoptosis inhibition in cancer cells: a novel molecular pathway that involves BAG3 protein. Int $J$ Biochem Cell Biol 2007; 39: 1337-1342.

3. Takayama S, Reed JC. Molecular chaperone targeting and regulation by BAG family proteins. Nat Cell Biol 2001; 3: 237-241.

4. Coulson M, Robert S, Saint R. Drosophila starvin encodes a tissue-specific BAG-domain protein required for larval food uptake. Genetics 2005; 171: 1799-1812.

5. Doukhanina EV, Chen S, van der Zalm E, Godzik A, Reed J, Dickman MB. Identification and functional characterization of the BAG protein family in Arabidopsis thaliana. J Bio Chem 2006; 281: 18793-18801.

6. Colinet $\mathrm{H}$, Hoffmann A. Gene and protein expression of Drosophila Starvin during cold stress and recovery from chill coma. Insect Biochem Mol Biol 2010; 40: 425-428.

7. Doong H, Rizzo K, Fang S, Kulpa V, Weissman AM, Kohn EC. CAIR-1/BAG-3 abrogates heat shock protein-70 chaperone complex-mediated protein degradation: accumulation of poly-ubiquitinated Hsp90 client proteins. J Biol Chem 2003; 278: 28490-28500.

8. Beere HM. Death versus survival: functional interaction between the apoptotic and stress-inducible heat shock protein pathways. J Clin Invest 2005; 115: 2633-2639.

9. Doong H, Vrailas A, Kohn EC. What's in the 'BAG'?-a functional domain analysis of the BAG-family proteins. Cancer Lett 2002; 188: 25-32.
10. Pagliuca MG, Lerose R, Cigliano S, Leone A. Regulation by heavy metals and temperature of the human BAG-3 gene, a modulator of Hsp70 activity. FEBS Lett 2003; 541: 11-13.

11. Bruno AP, Festa M, Dal Piaz F, Rosati A, Turco MC, Giuditta A et al. Identification of a synaptosome-associated form of BAG3 protein. Cell Cycle 2008; 7: 3104-3105.

12. Young $P$, Anderton $E$, Paschos K, White R, Allday MJ. Epstein-Barr virus nuclear antigen (EBNA) $3 A$ induces the expression of and interacts with a subset of chaperones and co-chaperones. J Gen Virol 2008; 89: 866-877.

13. Rosati A, Di Salle E, Luberto L, Quinto I, Scala G, Turco MC et al. Identification of a Btk-BAG3 complex induced by oxidative stress. Leukemia 2009; 23: 823-824.

14. Rosati A, Leone A, Del Valle L, Amini S, Khalili K, Turco MC. Evidence for BAG3 modulation of HIV-1 gene transcription. J Cell Physiol 2007; 210: 676-683.

15. Kyratsous CA, Silverstein SJ. The co-chaperone BAG3 regulates Herpes simplex virus replication. Proc Natl Acad Sci USA 2008; 105: 20912-20917.

16. Kyratsous CA, Silverstein SJ. BAG3, a host cochaperone, facilitates varicella-zoster virus replication. J Virol 2007; 81: 7491-7503.

17. Zhang L, Zhang ZP, Zhang XE, Lin FS, Ge F. Quantitative proteomics analysis reveals BAG3 as a potential target to suppress severe acute respiratory syndrome coronavirus replication. J Virol 2010; 84: 6050-6059.

18. Gout E, Gutkowska M, Takayama S, Reed JC, Chroboczek J. Co-chaperone BAG3 and adenovirus penton base protein partnership. J Cell Biochem 2010; 111: 699-708.

19. Rosati A, Khalili K, Deshmane SL, Radhakrishnan S, Pascale M, Turco MC et al. BAG3 protein regulates caspase-3 activation in HIV-1-infected human primary microglial cells. J Cell Physiol 2009; 218: 264-267.

20. Liao Q, Ozawa F, Friess H, Zimmermann A, Takayama S, Reed JC et al. The anti-apoptotic protein BAG-3 is overexpressed in pancreatic cancer and induced by heat stress in pancreatic cancer cell lines. FEBS Lett 2001; 503: 151-157.

21. Romano MF, Festa M, Pagliuca G, Lerose R, Bisogni R, Chiurazzi F et al. BAG3 protein controls B-chronic lymphocytic leukaemia cell apoptosis. Cell Death Differ2003; 10: 383-385.

22. Romano MF, Festa M, Petrella A, Rosati A, Pascale M, Bisogni R et al. BAG3 protein regulates cell survival in childhood acute lymphoblastic leukemia cells. Cancer Biol Ther 2003; 2: 508-510.

23. Homma S, Iwasaki M, Shelton GD, Engvall E, Reed JC, Takayama S. BAG3 deficiency results in fulminant myopathy and early lethality. Am J Pathol 2006; 169: 761-773.

24. Chiappetta G, Ammirante M, Basile A, Rosati A, Festa M, Monaco M et al. The antiapoptotic protein BAG3 is expressed in thyroid carcinomas and modulates apoptosis mediated by tumor necrosis factor-related apoptosis-inducing ligand. J Clin Endocrinol Metab 2007; 92: 1159-1163

25. Rosati A, Ammirante M, Gentilella A, Basile A, Festa M, Pascale M et al. Apoptosis inhibition in cancer cells: a novel molecular pathway that involves BAG3 protein. Int J Biochem Cell Biol 2007; 39: 1337-1342.

26. Ben Aicha S, Lessard J, Pelletier M, Fournier A, Calvo E, Labrie C. Transcriptional profiling of genes that are regulated by the endoplasmic reticulum-bound transcription factor AlbZIP/CREB3L4 in prostate cells. Physiol Genomics 2007; 31: 295-305.

27. Iwasaki M, Homma S, Hishiya A, Dolezal SJ, Reed JC, Takayama S. BAG3 regulates motility and adhesion of epithelial cancer cells. Cancer Res 2007; 67: 10252-10259.

28. Valdez BC, Murray D, Ramdas L, de Lima M, Jones R, Kornblau S et al. Altered gene expression in busulfan-resistant human myeloid leukemia. Leuk Res 2008; 32: 1684-1697.

29. Gentilella A, Passiatore G, Deshmane S, Turco MC, Khalili K. Activation of BAG3 by Egr-1 in response to FGF-2 in neuroblastoma cells. Oncogene 2008; 27: 5011-5018.

30. Liu P, Xu B, Li J, Lu H. BAG3 gene silencing sensitizes leukemic cells to Bortezomibinduced apoptosis. FEBS Lett 2009; 583: 401-406

31. Jacobs AT, Marnett LJ. HSF1-mediated BAG3 expression attenuates apoptosis in 4-hydroxynonenal-treated colon cancer cells via stabilization of anti-apoptotic Bcl-2 proteins. J Biol Chem 2009; 284: 9176-9183.

32. Kassis JN, Virador VM, Guancial EA, Kimm D, Ho AS, Mishra M et al. Genomic and phenotypic analysis reveals a key role for CCN1 (CYR61) in BAG3-modulated adhesion and invasion. J Pathol 2009; 218: 495-504.

33. Staibano S, Mascolo M, Di Benedetto M, Vecchione ML, Ilardi G, Di Lorenzo G et al. BAG3 protein delocalisation in prostate carcinoma. Tumour Biol 2010; 31: 461-469.

34. Wang $H Q$, Liu BQ, Gao YY, Meng X, Guan Y, Zhang HY et al. Inhibition of the JNK signalling pathway enhances proteasome inhibitor-induced apoptosis of kidney cancer cells by suppression of BAG3 expression. Br J Pharmacol 2009; 158: 1405-1412.

35. Chen L, Wu W, Dentchev T, Zeng Y, Wang J, Tsui I et al. Light damage induced changes in mouse retinal gene expression. Exp Eye Res 2004; 79: 239-247.

36. Festa M, Del Valle L, Franco R, Scognamiglio G, Khalili K, De Laurenzi L et al. BAG3 protein is overexpressed in human glioblastoma and is a potential target for its therapy. Am J Pathol 2011 (in press)

37. Ammirante M, Rosati A, Arra C, Festa M, Basile A, Falco A et al. IKK $\gamma$ protein is a target of BAG3 regulatory activity in human tumor growth. Proc Natl Acad Sci USA 2010; 107: 7497-7502.

38. Lee MY, Kim SY, Choi JS, Choi YS, Jeon MH, Lee JH et al. Induction of Bis, a Bcl-2-binding protein, in reactive astrocytes of the rat hippocampus following kainic acid-induced seizure. Exp Mol Med 2002; 34: 167-171.

39. Bonelli P, Petrella A, Rosati A, Romano MF, Lerose R, Pagliuca MG et al. BAG3 protein regulates stress-induced apoptosis in normal and neoplastic leukocytes. Leukemia 2004; 18: $358-360$. 
40. Tabuchi Y, Ando H, Takasaki I, Feril Jr LB, Zhao QL, Ogawa R et al. Identification of genes responsive to low intensity pulsed ultrasound in a human leukemia cell line Molt-4. Cancer Lett 2007; 246: 149-156.

41. Friedrich B, Janessa A, Schmieder R, Risler T, Alexander D. Acute effects of haemodialysis on pro-/anti- apoptotic genes in peripheral blood leukocytes. Cell Physiol Biochem 2008; 22: 423-430.

42. Jacobs AT, Marnett LJ. Systems analysis of protein modification and cellular responses induced by electrophile stress. Acc Chem Res 2010; 43: 673-683.

43. Basile A, Zeppa R, Pasquino N, Arra C, Ammirante M, Festa M et al. Exposure to $50 \mathrm{~Hz}$ electromagnetic field raises the levels of the anti-apoptotic protein BAG3 in melanoma cells. J Cell physiol 2011; e-pub ahead of print 1 February 2011.

44. Jung SE, Kim YK, Youn DY, Lim MH, Ko JH, Ahn YS et al. Down-modulation of Bis sensitizes cell death in $\mathrm{C} 6$ glioma cells induced by oxygen-glucose deprivation. Brain Res 2010; 1349: 1-10.

45. Voellmy R, Boellmann F. Chaperone regulation of the heat shock protein response. Adv Exp Med Biol 2007; 594: 89-99.

46. Dai $C$, Whitesell L, Rogers $A B$, Lindquist $S$. Heat shock factor 1 is a powerful multifaceted modifier of carcinogenesis. Cell 2007; 130: 1005-1018.

47. Franceschelli S, Rosati A, Lerose R, De Nicola S, Turco MC, Pascale M. Bag3 gene expression is regulated by heat shock factor 1 . J Cell Physiol 2008; 215: 575-577.

48. Song S, Kole S, Precht P, Pazin MJ, Bernier M. Activation of heat shock factor 1 plays a role in pyrrolidine dithiocarbamate-mediated expression of the co-chaperone BAG3. Int $J$ Biochem Cell Biol 2010; 42: 1856-1863.

49. Cesaro E, Montano G, Rosati A, Crescitelli R, Izzo P, Turco MC et al. WT1 protein is a transcriptional activator of the antiapoptotic bag3 gene. Leukemia 2010; 24: 1204-1206.

50. Yang L, Han Y, Suarez Saiz F, Minden MD. A tumor suppressor and oncogene: the WT1 story. Leukemia 2007; 21: 868-876.

51. Gentilella A, Khalili K. Autoregulation of co-chaperone BAG3 gene transcription. J Cell Biochem 2009; 108: 1117-1124.

52. Du ZX, Meng X, Zhang HY, Guan Y, Wang HQ. Caspase-dependent cleavage of BAG3 in proteasome inhibitors-induced apoptosis in thyroid cancer cells. Biochem Biophys Res Commun 2008; 369: 894-898

53. Wang HQ, Liu HM, Zhang HY, Guan Y, Du ZX. Transcriptional upregulation of BAG3 upon proteasome inhibition. Biochem Biophys Res Commun 2008; 365: 381-385

54. Choi JS, Lee JH, Kim HY, Chun MH, Chung JW, Lee MY. Developmental expression of Bis protein in the cerebral cortex and hippocampus of rats. Brain Res 2006; 1092: 69-78.

55. Gentilella A, Khalili K. BAG3 expression is sustained by FGF2 in neural progenitor cells and impacts cell proliferation. Cell Cycle 2010; 9: 4245-4247.

56. Choi JS, Lee JH, Shin YJ, Lee JY, Yun H, Chun MH et al. Transient expression of Bis protein in midline radial glia in developing rat brainstem and spinal cord. Cell Tissue Res 2009; 337: 27-36.

57. De Marco M, Turco MC, Rosati A. BAG3 protein is induced during cardiomyoblast differentiation and modulates myogenin expression. Cell Cycle 2011; 10: 850-852.

58. Selcen D, Muntoni F, Burton BK, Pegoraro E, Sewry C, Bite AV et al. Mutation in BAG3 causes severe dominant childhood muscular dystrophy. Ann Neurol 2009; 65: 83-89.

59. McCollum AK, Casagrande G, Kohn EC. Caught in the middle: the role of Bag3 in disease. Biochem J 2009; 425: 1-3.

60. Selcen D. Myofibrillar myopathies. Curr Opin Neurol 2010; 23: 477-481.

61. Kwon KR, Ahn JY, Kim MS, Jung JY, Lee JH, Oh IH. Disruption of bis leads to the deterioration of the vascular niche for hematopoietic stem cells. Stem Cells 2010; 28: 268-278.

62. Wang $H Q$, Meng X, Gao YY, Liu BQ, Niu XF, Zhang HY et al. Characterization of BAG3 cleavage during apoptosis of pancreatic cancer cells. J Cell Physiol 2010; 224 $94-100$

63. Lüders J, Demand J, Höhfeld J. The ubiquitin-related BAG-1 provides a link between the molecular chaperones Hsc70/Hsp70 and the proteasome. J Biol Chem 2000; 275 4613-4617.

64. Matsui $\mathrm{H}$, Asou $\mathrm{H}$, Inaba T. Cytokines direct the regulation of Bim mRNA stability by heatshock cognate protein 70. Mol Cell 2007; 25: 99-112.

65. Kassis JN, Guancial EA, Doong H, Virador V, Kohn EC. CAIR-1/BAG-3 modulates cell adhesion and migration by downregulating activity of focal adhesion proteins. Exp Cell Res 2006; 312: 2962-2971.

66. Fontanella B, Birolo L, Infusini G, Cirulli C, Marzullo L, Pucci $P$ et al. The co-chaperone BAG3 interacts with the cytosolic chaperonin CCT: new hints for actin folding. Int $J$ Biochem Cell Biol 2010; 42: 641-650.

67. Iwasaki M, Tanaka R, Hishiya A, Homma S, Reed JC, Takayama S. BAG3 directly associates with guanine nucleotide exchange factor of Rap1, PDZGEF2, and regulates cell adhesion. Biochem Biophys Res Commun 2010; 400: 413-418.

68. Gilmore AP. Anoikis. Cell Death Differ 2005; 12: 1473-1477.

69. Moreau K, Luo S, Rubinsztein DC. Cytoprotective roles for autophagy. Curr Opin Cell Biol 2010; 22: 206-211.

70. Carra S. The stress-inducible HspB8-Bag3 complex induces the elF2alpha kinase pathway: implications for protein quality control and viral factory degradation? Autophagy 2009; 5: 428-429.

71. Carra S, Brunsting JF, Lambert $\mathrm{H}$, Landry J, Kampinga $\mathrm{HH}$. HspB8 participates in protein quality control by a non-chaperone-like mechanism that requires elF2\}\{alphal\} phosphorylation. J Biol Chem 2009; 284: 5523-5532.

72. Fuchs M, Poirier DJ, Seguin SJ, Lambert H, Carra S, Charette SJ et al. Identification of the key structural motifs involved in HspB8/HspB6-Bag3 interaction. Biochem J 2009; 425 245-255.

73. Fan GC, Chu G, Kranias EG. Hsp20 and its cardioprotection. Trends Cardiovasc Med 2005; 15: 138-141.

74. Dreiza CM, Komalavilas P, Furnish EJ, Flynn CR, Sheller MR, Smoke CC et al. The small heat shock protein, HSPB6, in muscle function and disease. Cell Stress Chaperones 2010; 15: 1-11.

75. Gamerdinger M, Hajieva P, Kaya AM, Wolfrum U, Hartl FU, Behl C. Protein quality control during aging involves recruitment of the macroautophagy pathway by BAG3. EMBO J 2009; 28: 889-901.

76. Baud V, Karin M. NF-kappaB a good target for cancer therapy? Hopes and pitfalls. Nat Rev Drug Discov 2009; 8: 33-40.

77. Karin M. NF-kappaB as a critical link between inflammation and cancer. Cold Spring Harb Perspect Biol 2009; 1: a000141.

78. McAllister SS, Weinberg RA. Tumor-host interactions: a far-reaching relationship. J Clin Oncol 2010; 28: 4022-4028.

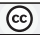

OMERIGHS

published by Nature Publishing Group. This work is under the Creative Commons Attribution-Noncommercial-No Derivative Works 3.0 Unported License. To view a copy of this license, visit http://creativecommons.org/licenses/by-nc-nd/3.0/ 